\title{
Elaboración de un alimento para ganado bovino a base de zanahoria (Daucus carota L.) mediante fermentación en estado sólido como una alternativa ecoeficiente
}

\section{Food preparation for carrot-based cattle (Daucus carota L.) using solid state fermentation for an eco-efficient alternative}
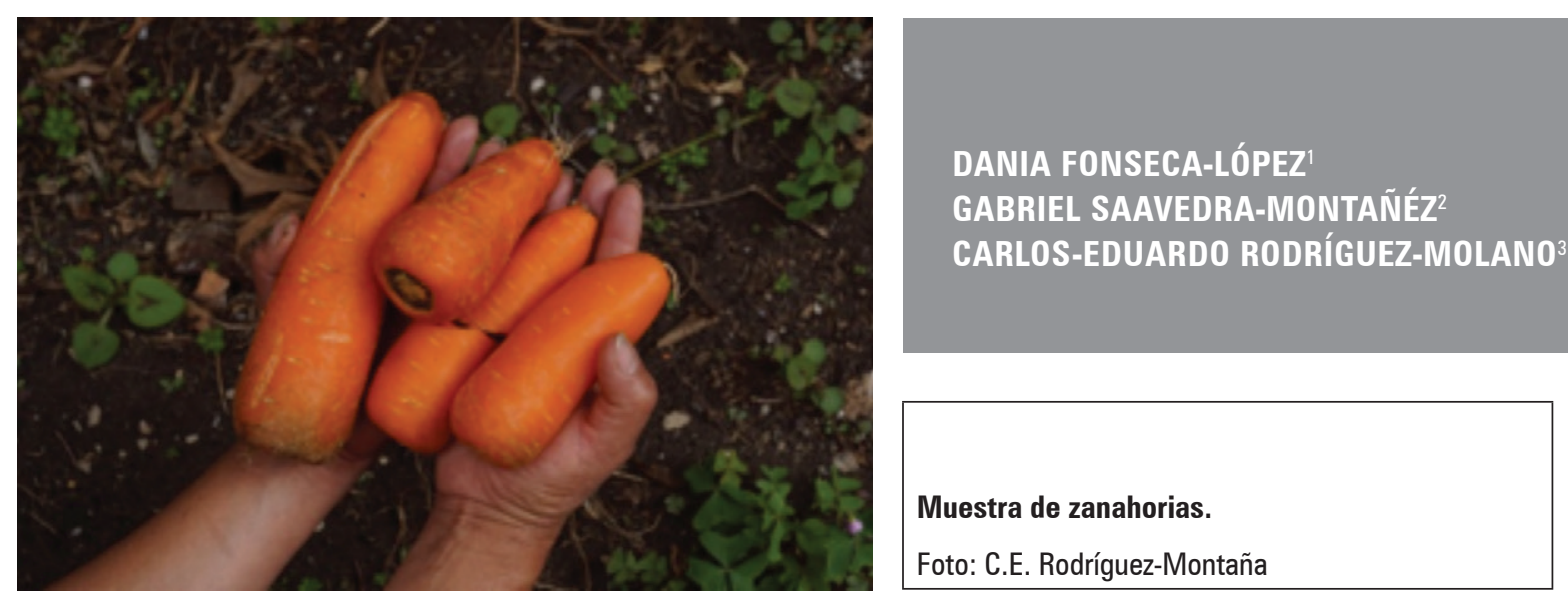

\section{RESUMEN}

La alimentación del ganado bovino debe basarse en estrategias sostenibles debido a la presión que se ejerce sobre los recursos naturales para la obtención de alimentos concentrados, una de dichas estrategias es la fermentación en estado sólido (FES), la cual, permite diseñar alimentos con menor impacto ambiental, debido a la acción de microorganismos eficientes que enriquecen sustratos con proteína de alto valor biológico. El objetivo de la investigación fue elaborar un alimento mediante FES a base de zanahoria (Daucus carota L.) en mezcla con otros ingredientes como alternativa para la alimentación de ganado, para ello, se obtuvieron tres productos fermentados en estado sólido con la inclusión de zanahoria (FES 1 [control], FES 2 [sin repila de trigo] y FES 3 [sin cascarilla de café]). Se evaluó la variación del pH durante el proceso de fermentación, se hizo análisis composicional y análisis microbiológico de los alimentos. Se obtuvo un aumento de cuatro veces el valor proteico (PB) inicial a las 48 horas pasando de $4,34 \%$ a $19 \%$ (FES 1), 17,4\% (FES 2) y 14,4\% (FES 3) $(P \leq 0,05)$, se observó el mayor crecimiento de mesófilos totales a las 24 horas con $69 \times 10^{-3} \mathrm{UFC} / \mathrm{g}$ (FES 1 ), $120 \times 10^{-3} \mathrm{UFC} / \mathrm{g}(\mathrm{FES} 2)$ y $45 \times 10^{-3} \mathrm{UFC} / \mathrm{g}$ (FES 3$)(P \leq 0,05)$; el $\mathrm{pH}$ descendió a las 96 horas de fermentación

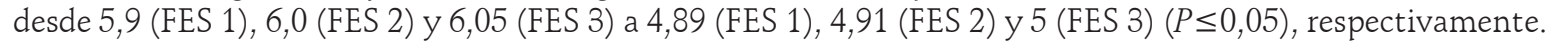
Se concluye que con el uso de la biotecnología FES es posible utilizar la zanahoria en mezcla con otras materias primas para la obtención de un alimento ecoeficiente para ganado.

Facultad de Ciencias Agropecuarias, Grupo de investigación GIBNA, Universidad Pedagógica y Tecnológica de Colombia, Tunja (Colombia). ORCID Fonseca-López, D.: 0000-0003-3877-3730

2 Facultad de Ciencias Agropecuarias, Grupo de investigación GIBNA, Universidad Pedagógica y Tecnológica de Colombia, Tunja (Colombia). Saavedra-Montañéz, G.: 0000-0003-0463-7722

3 Facultad de Ciencias Agropecuarias, Grupo de investigación GIBNA, Universidad Pedagógica y Tecnológica de Colombia, Tunja (Colombia). ORCID Rodríguez-Molano, C.E.: 0000-0002-0862-3478

4 Autor para correspondencia. carlos.rodriguez@uptc.edu.co 


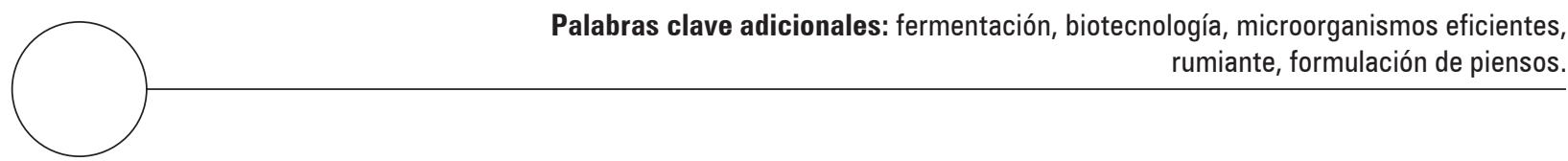

\begin{abstract}
Livestock feeding must use sustainable strategies because of the pressure exerted on natural resources seen with concentrated feed; solid state fermentation (FES) allows for the design of foods with less environmental impact because of the action of efficient microorganisms that enrich the residues of crops and flours with a low nutritional value. The objective of this research was to create a carrot-based FES (Daucus carota L.) food in combination with other ingredients as an alternative for cattle feed; for this, three solid-state fermented products ( 72 hours) were obtained with the inclusion of carrot (FES 1 [control], FES 2 [without repel of wheat] and FES 3 [without coffee husk]). The $\mathrm{pH}$ variation was evaluated during the fermentation process, and a compositional analysis and microbiological analysis of the diets were performed. A 4-fold increase in the initial protein value (BP) at 48 hours was obtained, from 4.34 to $19 \%$ (FES 1), 17.4\% (FES 2) and 14.4\% (FES 3) $(P \leq 0.05)$. The highest growth of total mesophiles at 24 hours was observed with $69 \times 10^{-3} \mathrm{UFC} / \mathrm{g}$ (FES 1), $120 \times 10^{-3} \mathrm{UFC} / \mathrm{g}$ (FES 2) and $45 \times 10^{-3} \mathrm{UFC} / \mathrm{g}$ (FES 3) $(P \leq 0,05)$; the pH dropped at 96 hours of fermentation of 5.9 (FES 1) (FES 1), 6 (FES 2) and 6.05 (FES 3) at 4.89 (FES 1), 4.91 (FES 2) and 5 (FES 1) FES 3) (P $\leq 0.05$ ), respectively. It was concluded that, with the FES biotechnology, it is possible to use carrot mixed with other raw materials to obtain eco-efficient cattle feed.
\end{abstract}

Additional key words: fermentation, biotechnology, efficient microorganisms, ruminant, feed formulation.

Fecha de recepción: 24-10-2017 Aprobado para publicación: 30-01-2018

INTRODUCCIÓN

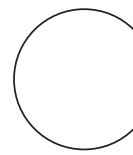

El departamento de Boyacá se encuentra dentro de los principales productores de zanahoria (Daucus carota L.) con un área cultivada de 1.765 ha, rendimientos de 38,13 t ha-1 (Agronet, 2015) y pérdidas del $1 \%$ asociadas al tamaño reducido, deformaciones y alteraciones en la superficie de la hortaliza. Esto último, no solo genera pérdidas sino un subproducto de bajo valor nutricional que puede ser utilizado como alimento alternativo para bovinos mediante la fermentación en estado sólido (FES) siendo esta una tecnología que posibilita el enriquecimiento de sustratos a partir de inoculantes, los cuales, son microrganismos que utilizan ha dicho sustrato como fuente de nutrientes y anclaje (Biz et al., 2016) y a cambio, aportan proteína de alto valor biológico y energía. A raíz de ello, el objetivo de este trabajo fue elaborar un alimento FES a base de zanahoria como alternativa ecoeficiente para la alimentación de ganado en mezcla con otras materias primas de fácil adquisición en la zona como la tuza de mazorca Zea maiz L. (Poaceae), el nabo Tropaeolum tuberosum
Ruiz \& Pav. (Tropaeolaceae) y la morera Morus alba L. (Moraceae).

\section{MATERIALES Y MÉTODOS}

\section{Localización}

Este trabajo se desarrolló en el laboratorio de nutrición animal y en el Laboratorio de Fisiología Vegetal de la Universidad Pedagógica y Tecnológica de Colombia (UPTC), seccional Tunja.

\section{Tratamientos}

Se compararon tres alimentos, un control (FES 1) que correspondió a un alimento base (35\% de zanahoria) a partir del cual se diseñaron dos adicionales, en estos se sustituyó los materiales secantes cascarilla de café y repila de trigo por una mezcla de nabo, morera y 
tusa de mazorca, de tal manera, que las dietas fueron isoproteicas e isoenergéticas según tablas del National Research Council (NCR) formando un FES 2 sin repila de trigo y FES 3 sin cascarilla de café (Tab. 1).

\section{Tabla 1. Composición porcentual de tres alimentos evaluados.}

\begin{tabular}{|l|c|c|c|}
\hline \multicolumn{1}{|c|}{ Materia prima } & FES 1 & FES 2 & FES 3 \\
\hline Nabo & 0 & 17,5 & 5,2 \\
\hline Tusa & 0 & 2,4 & 3,8 \\
\hline Morera & 0 & 5 & 6 \\
\hline Cascarilla de café & 15 & 10,1 & 0 \\
\hline Repila de trigo & 20 & 0 & 20 \\
\hline Torta de palmiste (alta grasa) & 20 & 20 & 20 \\
\hline Zanahoria & 35 & 35 & 35 \\
\hline Sulfato de magnesio & 0,5 & 0,5 & 0,5 \\
\hline Premezcla vitamínica & 0,5 & 0,5 & 0,5 \\
\hline Inoculante Liofast ${ }^{\circledR}$ & 2 & 2 & 2 \\
\hline Melaza & 5 & 5 & 5 \\
\hline Urea & 1,5 & 1,5 & 1,5 \\
\hline Carbonato de calcio & 0,5 & 0,5 & 0,5 \\
\hline Total & 100 & 100 & 100 \\
\hline
\end{tabular}

Los ingredientes utilizados se caracterizaron por tener un bajo contenido de proteína (Tab. 2) a excepción de la morera y el ryegrass (Lolium multiflorum Lam. (Poaceae)) cuyos contenidos de proteína bruta (PB) y fibra detergente neutra (FDN) los ubican como alimentos de alta calidad al contener más de 15\% de PB y menos de 50\% de FDN (Di Marco, 2011).
Por otro lado, la zanahoria utilizada se caracterizó por tener una firmeza de 115,9 $\pm 0,10 \mathrm{~N}$ (Newton), $\mathrm{pH}$ $6,79 \pm 0,01\left(18^{\circ} \mathrm{C}\right)$ y un contenido promedio de sólidos solubles totales (SST) de $16,63 \pm 0,07^{\circ} \mathrm{Brix}$, mientras que el nabo obtuvo una firmeza de $35,18 \pm 0,01$ $\mathrm{N}, \mathrm{pH} 7,11 \pm 0,01\left(18^{\circ} \mathrm{C}\right)$ y un contenido de SST de $6,25 \pm 0,07^{\circ}$ Brix.

\section{Preparación del alimento mediante FES}

Los ingredientes sólidos: nabo y zanahoria se picaron en tamaño de $4 \mathrm{~mm}$ aproximadamente, mientras que la tusa y el material vegetal de morera se secaron y se pasaron por una máquina pica pasto marca Indutornometal JR ${ }^{\circledR}$ (Funza, Colombia) hasta la presentación de harina. Todos los ingredientes se mezclaron homogéneamente y se inocularon con un preparado microbiano a partir de la metodología propuesta por Borras-Sandoval et al. (2015) utilizando cultivo comercial Liofast Y452B, SACCO ${ }^{\circledR}$ (Cadorago, Italia). Luego, las mezclas se empacaron en bolsas plásticas herméticas con capacidad de $25 \mathrm{~kg}$ según el tratamiento y se fermentaron en incubadora a $20^{\circ} \mathrm{C}$ por $72 \mathrm{~h}$

\section{Variables evaluadas}

Análisis composicional. Se determinó el contenido de materia seca (MS) con una estufa de secado marca Memmert $^{\circledR}$ (Karl Kolb, Schwabach, Alemania) y humedad $(\mathrm{H})$ por diferencia; cenizas (Cen) por método de incineración a $550^{\circ} \mathrm{C}$ con mufla Thermolyne ${ }^{\circledR}$ (Dubuque, IA); proteína bruta (PB) por el método de Kjeldahl; extracto etéreo (EE) por soxhlet; fibra cruda (FC), fibra en detergente ácida (FDA) y fibra en

\begin{tabular}{|c|c|c|c|c|c|c|c|c|c|}
\hline \multicolumn{2}{|c|}{ Materia prima } & H & MS & FDN & FDA & EE & Cen & PB & FC \\
\hline \multirow{7}{*}{$\begin{array}{l}\text { Ingredientes } \\
\text { FES }\end{array}$} & Tusa & 46,6 & 53,3 & 70,1 & 5,3 & 1 & 3,6 & 0,3 & 14,4 \\
\hline & Nabo & 27,2 & 72,1 & 54,9 & 15,9 & 1,6 & 7,3 & 2,1 & 5 \\
\hline & $\begin{array}{l}\text { Torta de palmiste } \\
\text { (alta grasa) }\end{array}$ & 8,6 & 91,3 & 78,2 & 13,8 & 8,4 & 2,8 & 8 & 0,8 \\
\hline & Zanahoria & 21,7 & 78,3 & 55,9 & 19,5 & 5,1 & 7,1 & 1,4 & 8,2 \\
\hline & Repila de trigo & 3,5 & 96,4 & 75,4 & 13,7 & 1,4 & 6,1 & 8,4 & 17,2 \\
\hline & Cascarilla café & 7,2 & 92,7 & 79,4 & 13,5 & 1,3 & 0,5 & 5,6 & 17,8 \\
\hline & Morera & 65,4 & 34,6 & 40,3 & 7,7 & 0,8 & 8,6 & 14,6 & 18,9 \\
\hline \multicolumn{2}{|l|}{ Ryegrass } & 77,8 & 22,2 & 38,9 & 6,5 & 1,6 & 11,4 & 23,1 & 17,2 \\
\hline
\end{tabular}

H: humedad; MS: materia seca; FDN: fibra detergente neutro; FDA: fibra detergente ácida; EE: extracto etéreo; Cen: cenizas; PB: proteína bruta; FC: fibra cruda. 
detergente neutra (FDN) por el método Van Soest et al. (1991).

pH durante el proceso de fermentación. Se evaluó el $\mathrm{pH}$ de las dietas fermentadas con un potenciómetro $\mathrm{OAKTON}^{\circledR}$ (OAKTON Instruments, Vernon Hills, IL, USA) desde el inicio hasta las $96 \mathrm{~h}$, a partir de un filtrado obtenido de una muestra de $5 \mathrm{~g}$ de alimento mezclado en $45 \mathrm{~mL}$ de agua destilada según el método de Elías et al. (1990).

Análisis microbiológico. Se utilizó el método recuento en placa NTC 4092 utilizando dilución $10^{-3}$, el cultivo se realizó con los procedimientos Compact Dry: E. coliformes ${ }^{\circledR}$, aerobios mesófilos ${ }^{\circledR}$, mohos y levaduras ${ }^{\circledR}$, incubados a $37^{\circ} \mathrm{C}$ por $48 \mathrm{~h}$ hasta las $72 \mathrm{~h}$ de fermentación de las dietas.

\section{Análisis estadístico}

Para el análisis estadístico se aplicó la prueba de homogeneidad de Levene, la prueba de normalidad por Shapiro willk, análisis de varianza (Anova) y comparación de promedios por Tukey $(P \leq 0,05)$.

\section{RESULTADOS Y DISCUSIÓN}

\section{Análisis composicional}

Se encontraron diferencias en las variables $\mathrm{MS} \%, \mathrm{H} \%$, $\mathrm{EE} \%, \mathrm{FDA} \%, \mathrm{FC} \%, \mathrm{~PB} \%$, Cen\% entre las dietas: $\mathrm{FES}$ 1 (control), FES 2 (sin repila de trigo) y FES 3 (sin cascarilla de café) (Tab. 3).

\section{Humedad $(\mathrm{H})$}

$\mathrm{La} \mathrm{H}$ es uno de los principales factores que influyen en la preparación del FES, puesto que el agua funciona como vehículo de los nutrientes e interviene en el crecimiento de la microbiota fermentadora tras involucrarse en sus procesos metabólicos. El nivel de $\mathrm{H}$ aumentó durante las horas de fermentación, siendo mayor en el FES 1 (Control) pasando de 44,3\% a las $24 \mathrm{~h}$ a $51,1 \%$ a las $72 \mathrm{~h}$, sin embargo, solo se presentaron diferencias estadísticas significativas a las 72 horas de fermentación en los tres alimentos $(P \leq 0,05)$. Este comportamiento está documentado por varios autores (Moyano, 2014; Borras-Sandoval et al., 2015), y se asocia a la producción de agua en los procesos metabólicos y a la formación de enlaces peptídicos en los que se pierde una molécula de agua por enlace formado, lo que explica el aumento del nivel de PB en los alimentos FES post-fermentación.

\section{Proteína bruta (PB)}

La proteína de los alimentos paso de 4,34\%, valor al que fueron balanceados los tres tratamientos, a 18,4\% en FES 1 (Control), 15,9\% en FES 2 (sin repila de trigo) y $18,3 \%$ FES 3 (sin cascarilla de café) luego de 24 $h$, lo que indica un aumento en promedio de cuatro veces el valor inicial con diferencias estadísticas a las 48 y $72 \mathrm{~h}$ de fermentación $(P \leq 0,05)$. Resultados similares fueron obtenidos por Díaz et al. (2014) en un alimento FES fermentado durante 20 días alcanzando una proteína de $15,83 \%$. Este comportamiento se relaciona con el aporte de nitrógeno (urea) que, según

Tabla 3. Análisis composicional (\%) de tres dietas usando zanahoria.

\begin{tabular}{|c|c|c|c|c|c|c|c|c|}
\hline $\begin{array}{c}\text { Horas de } \\
\text { fermentación }\end{array}$ & $\mathrm{T}$ & MS & $\mathrm{H}$ & EE & FDA & FC & PB & Cen \\
\hline \multirow{3}{*}{$24 \mathrm{~h}$} & FES 1 & $55,7 \pm 1,45 \mathrm{~ns}$ & $44,3 \pm 1,45 \mathrm{~ns}$ & $12,1 \pm 0,03 \mathrm{~b}$ & $44,6 \pm 0,95 \mathrm{~ns}$ & $40,5 \pm 0,49 \mathrm{~b}$ & $18,4 \pm 0,8 \mathrm{~ns}$ & $7,8 \pm 0,75 \mathrm{a}$ \\
\cline { 2 - 9 } & FES 2 & $53,9 \pm 2,26 \mathrm{~ns}$ & $46,1 \pm 2,26 \mathrm{~ns}$ & $15,2 \pm 0,11 \mathrm{a}$ & $56,7 \pm 1,58 \mathrm{~ns}$ & $48,2 \pm 0,26 \mathrm{a}$ & $15,9 \pm 0,1 \mathrm{~ns}$ & $6,5 \pm 0,10 \mathrm{~ns}$ \\
\cline { 2 - 9 } & FES 3 & $58,8 \pm 0,26 \mathrm{~ns}$ & $41,1 \pm 0,26 \mathrm{~ns}$ & $8,5 \pm 0,03 \mathrm{c}$ & $45,3 \pm 0,87 \mathrm{~ns}$ & $24,8 \pm 2,48 \mathrm{c}$ & $18,3 \pm 0,75 \mathrm{~ns}$ & $4,9 \pm 0,10 \mathrm{~b}$ \\
\hline \multirow{4}{*}{$48 \mathrm{~h}$} & FES 1 & $51,9 \pm 0,83 \mathrm{~ns}$ & $48 \pm 0,83 \mathrm{~ns}$ & $14,8 \pm 0,23 \mathrm{a}$ & $30,4 \pm 1,33 \mathrm{~b}$ & $34,7 \pm 1,02 \mathrm{~ns}$ & $19,1 \pm 0,5 \mathrm{a}$ & $7,5 \pm 0,15 \mathrm{a}$ \\
\cline { 2 - 9 } & FES 2 & $53,9 \pm 3,03 \mathrm{~ns}$ & $46,1 \pm 3,03 \mathrm{~ns}$ & $8,1 \pm 0,04 \mathrm{~b}$ & $42,6 \pm 0,37 \mathrm{a}$ & $39,1 \pm 1,82 \mathrm{~ns}$ & $17,4 \pm 0,1 \mathrm{a}$ & $6,4 \pm 0,10 \mathrm{~b}$ \\
\cline { 2 - 9 } & FES 3 & $59,7 \pm 1,69 \mathrm{~ns}$ & $40,3 \pm 1,69 \mathrm{~ns}$ & $12,8 \pm 0,18 \mathrm{a}$ & $39,9 \pm 0,43 \mathrm{~ns}$ & $36,8 \pm 0,35 \mathrm{~ns}$ & $14,3 \pm 0,55 \mathrm{~b}$ & $4,6 \pm 0,10 \mathrm{c}$ \\
\hline \multirow{3}{*}{$72 \mathrm{~h}$} & FES 1 & $48,9 \pm 0,64 \mathrm{c}$ & $51,1 \pm 0,64 \mathrm{a}$ & $4,1 \pm 0,01 \mathrm{~b}$ & $26,2 \pm 1,08 \mathrm{~ns}$ & $15,7 \pm 1,39 \mathrm{c}$ & $16,9 \pm 0,05 \mathrm{~b}$ & $7,5 \pm 0,25 \mathrm{ab}$ \\
\cline { 2 - 9 } & FES 2 & $51,7 \pm 1,78 \mathrm{~b}$ & $48,3 \pm 1,78 \mathrm{~b}$ & $3,5 \pm 0,01 \mathrm{~b}$ & $38,4 \pm 0,69 \mathrm{~ns}$ & $59,4 \pm 0,49 \mathrm{a}$ & $16,6 \pm 0,1 \mathrm{~b}$ & $5,3 \pm 0,10 \mathrm{~b}$ \\
\cline { 2 - 9 } & FES 3 & $58,1 \pm 1,02 \mathrm{a}$ & $41,9 \pm 1,02 \mathrm{c}$ & $21,3 \pm 0,02 \mathrm{a}$ & $30,5 \pm 0,59 \mathrm{~ns}$ & $33,7 \pm 0,96 \mathrm{~b}$ & $14,3 \pm 0,05 \mathrm{a}$ & $4,4 \pm 0,10 \mathrm{~b}$ \\
\hline
\end{tabular}

T: tratamiento, H: humedad, MS: materia seca, FDN: fibra detergente neutro, FDA: fibra detergente ácida, EE: extracto etéreo, Cen: cenizas, PB: proteína bruta, FC: fibra cruda.

Promedios con letras distintas indican diferencia significativa, entre dietas para cada tiempo de muestreo, según la prueba de Tukey $(P \leq 0,05) ; \pm$ error estándar; ns: sin diferencias estadísticas; a: $P \leq 0,05 ; \mathrm{b}: P \leq 0,01$. 
Brea-Maure et al. (2015) es utilizado junto con los carbohidratos fácilmente fermentables en la formación de protoplasma celular, aunque no se descarta una condensación de la proteína por la reducción de la MS. Esto explica a la vez, el menor contenido de PB en el FES 3 cuyo contenido de MS fue mayor al final del ensayo, de modo que en este alimento no se presentó un uso eficiente de nitrógeno ni de los carbohidratos para la formación de proteína de origen microbiano.

\section{Materia seca (MS)}

El contenido de MS se redujo con el tiempo de fermentación en los tratamientos, pasando de $55,7 \%$ (24 h) a 48,9\% (72 h) en el FES 1 (Control); de 53,9\% (24 h) a $51,7 \%$ (72 h) en el FES 2 (sin repila de trigo); y de $58,8 \%(24 \mathrm{~h})$ a $58,1 \%(72 \mathrm{~h})$ en el FES 3 (sin cascarilla de café) con diferencias estadísticas a las $72 \mathrm{~h}$ $(P \leq 0,05)$. Esto se relaciona con la utilización de los carbohidratos solubles como fuentes energéticas de los procesos metabólicos (Rodríguez et al., 2001) de los microrganismos presentes en el material, de modo que el contenido de carbono del sustrato soporte se consume con el tiempo de fermentación junto con las fuentes de nitrógeno y minerales.

\section{Cenizas (Cen)}

Los valores más altos se observaron en el FES 1 (Control) con 7,8\% (24 h), 7,5\% (48 h) y 7,5\% (72 h); valores intermedios en FES 2 (sin repila de trigo) con $6,5 \%$ (24 h), 6,4\% (48 h), 5,3\% (72 h); y los valores más bajos en FES 3 (sin cascarilla de café) con 4,9\% (24 h), 4,6\% (48 h), 4,4\% (72 h) existiendo diferencias estadísticas a las $24 \mathrm{~h}$ entre FES 1 y FES 3 ; y a las 48 y $72 \mathrm{~h}$ de fermentación $(P \leq 0,05)$ entre todos los tratamientos.

Este comportamiento se atribuye al uso de minerales como P, Ca, Mg, Fe y S en la formación de estructuras bacterianas y su intervención como cofactores en los procesos metabólicos. Sin embargo, la variación en el contenido de cenizas en los tratamientos también se relaciona con el contenido de cenizas de las fuentes energéticas usadas (Ramos et al., 2006).

\section{Fibra en detergente ácida (FDA)}

La FDA se redujo con el tiempo, sin embargo, solo hubo diferencias estadísticas $(P \leq 0,05)$ a las $48 \mathrm{~h}$ de fermentación entre el FES 1 (30,4\%) y el FES 2
$(42,6 \%)$. No obstante, el menor valor para FDA lo obtuvo el FES 1 (Control) a las $72 \mathrm{~h}$ con $26,2 \%$, resultado similar a lo reportado por Ramos et al. (2006) en la preparación del alimento FES sachacítrico con $25,82 \%$ y al FES sachapulido con $21,47 \%$. Esto indica una mejora en la digestibilidad del producto (Borras-Sandoval et al., 2014), debido a que la FDA y la digestibilidad están inversamente relacionados (Hernández, 2010) por lo que se concluye que las dietas a las $72 \mathrm{~h}$ tienen una mejor digestibilidad.

Fibra cruda (FC). Se observó diferencias estadísticas a las 24 y 72 h de fermentación en el contenido de FC de los tratamientos $(P \leq 0,05)$, el valor más alto fue para el FES 2 (sin repila de trigo) a las $72 \mathrm{~h}$ con $59,9 \%$ FC, seguido del FES 3 con $33,7 \%$ ( $P \leq 0,05)$, lo que puede estar relacionado con la fibra aportada por la morera cuya inclusión fue del 11\% para este tratamiento, mientras que en el FES 3 (sin cascarilla de café) la inclusión fue del $6 \%$.

Lo anterior indica que la inclusión de ingredientes con fibra vegetal suele favorecer el contenido de FC, y esto es muy importante en la prevención de los trastornos metabólicos (Hernández, 2010). Aunque también, dicho aumento en los valores de la FC según Aranda et al. (2012) se relaciona con un incremento significativo en el contenido de paredes celulares, en relación con el contenido de almidón del producto utilizado.

Sin embargo, en el FES 1 hubo una reducción en el contenido de FC pasando de 40,5\% (24 h) a 15,7\% (72 h) lo que se asocia con la actividad de microorganismos, debido a que estos pueden utilizar la hemicelulosa y parte del nitrógeno asociado a la fibra como fuente de nutrientes para crecer en los sustratos, antes de comenzar a degradar la lignina (Van Soest et al., 1991).

\section{Extracto etéreo (EE)}

Esta variable corresponde al grupo de nutrientes llamados grasa bruta o lípidos (Serna y López, 2010) donde también encontramos las vitaminas liposolubles. Se observó una reducción gradual conforme avanzo la fermentación desde las 24 hasta las $72 \mathrm{~h}$ pasando de 12,1 a 4,1\% en FES 1 (Control) y de 15,2 a 3,5\% FES 2 (sin repila de trigo) $(P \leq 0,05)$, debido a que las bacterias lácticas (BAL), tales como los géneros Streptococcus, Lactobacillus, Leuconostoc y algunos otros se destacan por sus requerimientos vitamínicos complejos (Madigan et al., 2004) y requerimientos de energía 
en forma de lípidos para la formación de enzimas, membranas y como fuentes de energía.

No obstante, se observó un efecto contrario en el FES 3 (sin cascarilla de café) con valores de $8,5 \%$ ( $24 \mathrm{~h}$ ), $12,8 \%$ (48 h) y $21,3 \%$ (72 h) $(P \leq 0,05)$, lo que se debe a una posible concentración de nutrientes debido a la reducción de los carbohidratos en el proceso fermentativo los cuales se utilizan como fuente energética, no solo por las BAL, sino también por la flora natural presente al inicio de la fermentación (Ortega y Hoyos, 2016).

\section{Análisis microbiológicos}

El crecimiento máximo de bacterias lácticas mesófilas se observó a las 24 h de fermentación en todos los tratamientos así: $120 \times 10^{-3} \mathrm{UFC} / \mathrm{g}$ (FES 2), $69 \times 10^{-3} \mathrm{UF}-$ $\mathrm{C} / \mathrm{g}$ (FES 1) y $45 \times 10^{-3} \mathrm{UFC} / \mathrm{g}$ (FES 3$)(P \leq 0,05)$. Con el paso de las horas se redujo las UFC de mesófilos, directamente por el consumo de carbohidratos para la formación de proteína bacteriana.

Aunque, el considerable crecimiento de UFC de mesófilos en las dietas FES 1 y FES 2 también se asocia con el pH más bajo y con el contenido de humedad de dichas dietas, debido a que el agua disuelve homogéneamente los nutrientes del medio y facilita su acceso a la célula, además de disolver los productos del metabolismo cuando se excretan al medio (Carrasco et al., 2003) (Fig. 1).

Sin embargo, el pH más bajo relacionado con la presencia de ácido láctico limitó el crecimiento de UFC de enterobacterias en las dietas FES 1 y FES 2 $(P \leq 0,05)$, pero en la dieta FES 3 se observó lo contrario, lo que se pudiera asociar al $\mathrm{pH}$ y humedad inicial que afecto el crecimiento de bacterias, entre las que se destacan las ácido lácticas y las mesófilas, y con ello la producción de ácido láctico capaz de ejercer un efecto antibiótico sobre los agentes microbianos no deseables (Fig. 2). En cuanto al crecimiento de UFC de mohos y levaduras, no se observaron diferencias estadísticas entre tratamientos, de modo que los valores estuvieron entre 0 y 3 UFC/g con una tendencia decreciente (Fig. 3).

Lo anterior por el aporte de nitrógeno (1,5\% urea) debido a que este ejerce un efecto inhibitorio en el crecimiento de hongos, así, la urea como fuente de nitrógeno en concentraciones desde el $1 \%$ frena el proceso de crecimiento para dar paso a una mayor esporulación (Agamez et al., 2008). Estos resultados demuestran que los alimentos FES son medios complejos

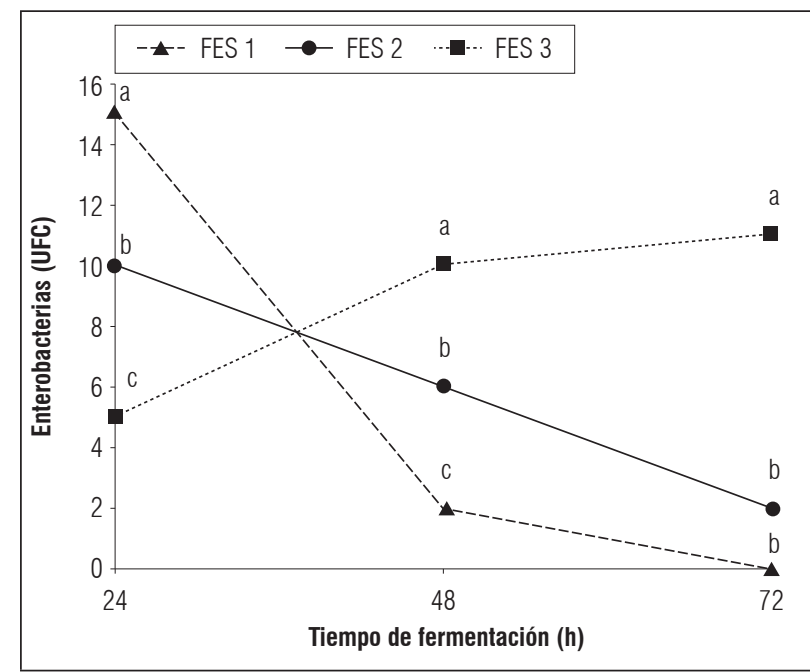

Figura 1. Crecimiento de mesófilos totales (UFC/g) en dietas fermentadas con zanahoria. Promedios con letras distintas indican diferencia significativa, en cada punto de muestreo, según la prueba de Tukey $(P \leq 0,05)$; ns: sin diferencias estadísticas.

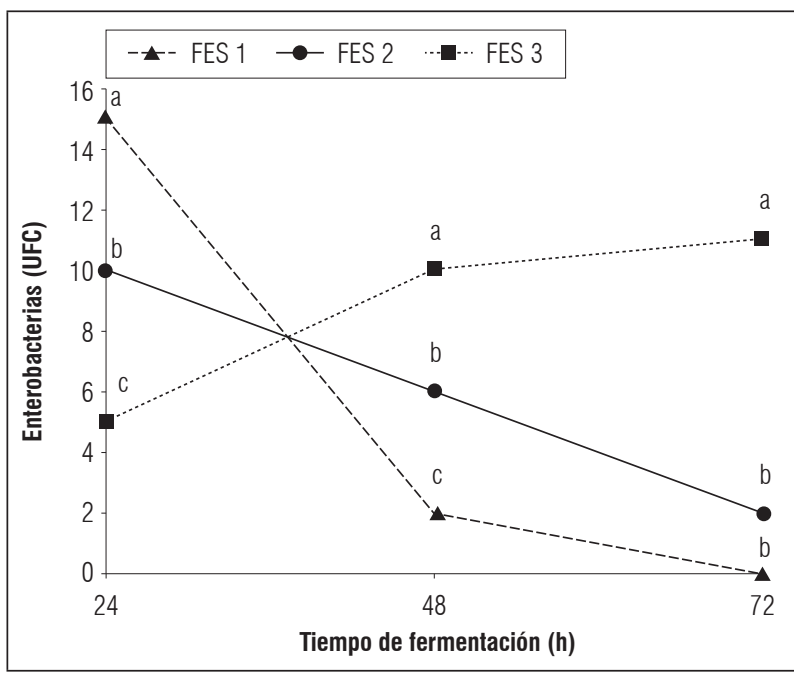

Figura 2. Crecimiento de enterobacterias (UFC/g) en dietas fermentadas con zanahoria. Promedios con letras distintas indican diferencia significativa, en cada punto de muestreo, según la prueba de Tukey $(P \leq 0,05)$.

que aportan nutrientes y favorecen el crecimiento de ciertos microorganismos, siendo el nivel de humedad un factor decisivo que influye en el proceso de fermentación pudiendo inactivar las bacterias lácticas cuando es bajo y con ello favorecer las condiciones para que se multipliquen organismos indeseables. 


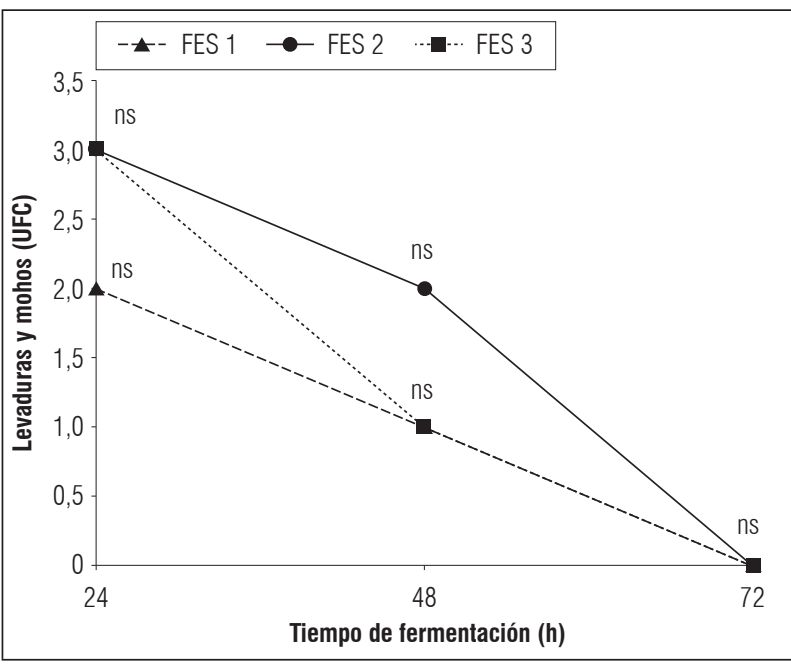

Figura 3. Crecimiento de mohos y levaduras (UFC/g) en dietas fermentadas con zanahoria. ns: sin diferencias estadísticas.

\section{pH durante el proceso de fermentación}

El $\mathrm{pH}$ es un indicador de la acidificación del medio y debe mantenerse entre 3,5 y 6,0 (Elías et al., 1990) para favorecer el crecimiento de bacterias mesófilas. Esto explica, porqué en el FES 3 (sin repila de café) no hubo un buen crecimiento de dichas bacterias al quedar preparado con un $\mathrm{pH}$ inicial de 6,05 mientras que en el FES 1 (Control) y en el FES 2 (sin repila de trigo) el crecimiento de UFC de mesófilos fue mayor (Fig. 4).

Se obtuvo valores iniciales de 5,9 (FES 1), 6 (FES 2) y 6,05 (FES 3) y finales de 5 en el FES 3, 4,89 en FES 1 y $4,91 \mathrm{FES} 2(P \leq 0,05)$. Estos resultados son similares a los encontrados por Borras-Sandoval et al. (2015) en el fes-papa con 4,86; pero mayores al rango reportado por Díaz et al. (2014) de 4,00 a 4,86 como óptimos en este proceso, por lo que se concluye que el nivel utilizado de carbonato de calcio $(0,5 \%)$ fue muy alto y esto a su vez influyo en el crecimiento bacteriano.

Lo que se debe, según Díaz-Plascencia et al. (2010) a que el calcio tiene propiedades bufferantes que mantienen el $\mathrm{pH}$ superior a 6 , lo que posiblemente deje fuera de rango la actividad microbial de las levaduras tal como lo observó en un proceso de fermentación con manzana. Igualmente, no se descarta que el nivel de urea también haya sido alto $(1,5 \%)$, debido a que una mayor concentración de la misma es capaz de aumentar el amoníaco y el pH (Ramos et al. 2006), lo que depende, en menor o mayor grado, de

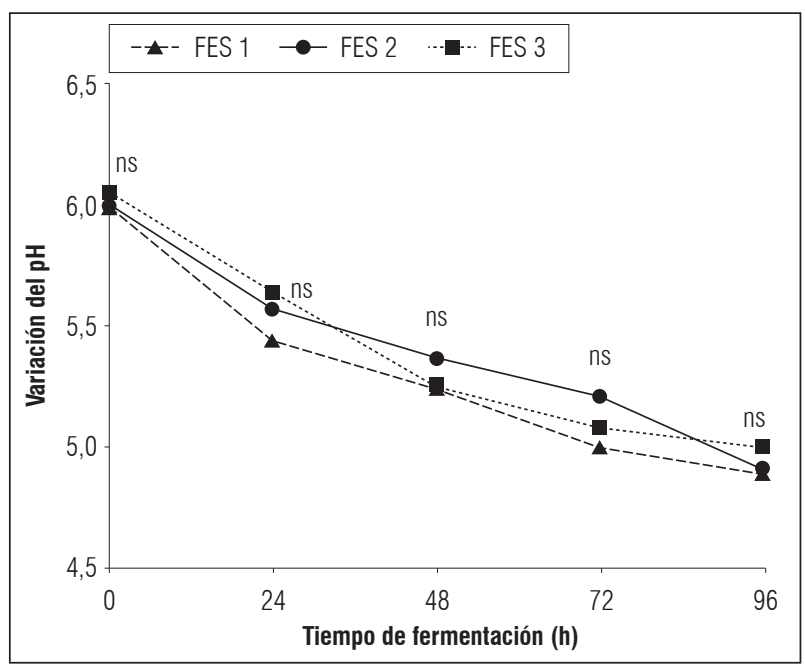

Figura 4. Variación del pH en dietas fermentadas con zanahoria. ns: sin diferencias estadísticas.

la concentración de los ácidos orgánicos (AGCC y láctico) (Brea-Maure et al., 2015) por lo que se sugiere utilizar concentraciones menores para próximos estudios.

\section{CONCLUSIONES}

La fermentación en estado sólido es un proceso donde microrganismos eficientes usan el sustrato componente del alimento para multiplicarse y realizar sus procesos metabólicos mediante el consumo de carbohidratos (MS), minerales (Cen), lípidos (EE) y nitrógeno aportado por la urea y la fibra cuando existe un medio favorable.

Factores como la humedad impactan el proceso de fermentación, en este trabajo se observó que una humedad inicial en el alimento cercana a 41,1\% (FES 3) reduce la disolución de nutrientes lo que limita el crecimiento de bacterias ácido lácticas. Además, niveles de $0,5 \%$ de carbonato de calcio y $1,5 \%$ de urea en el alimento aumentan el $\mathrm{pH}$ inicial dejándolo en un rango entre 5,9 y 6,05 , lo que frena el crecimiento de bacterias benéficas.

La zanahoria como alimento base en los alimentos FES es un sustrato sólido que aporta azucares deseables para el desarrollo de la microbiota fermentadora, además, es posible utilizarla en mezcla con otros materiales gracias a la capacidad fermentativa de los microorganismos eficientes obteniéndose proteínas de 16,17 y $16,6 \%$ (FES 2) comparables con las aportadas por alimentos comerciales. 


\section{AGRADECIMIENTOS}

Los autores manifiestan sus agradecimientos a la Universidad Pedagógica y Tecnológica de Colombia, al grupo GIBNA y a otras personas cuya colaboración fue imprescindible en la ejecución de este proyecto.

Conflicto de intereses: el manuscrito fue preparado y revisado con la participación de los autores, quienes declaran no tener algún conflicto de interés que coloquen en riesgo la validez de los resultados aquí presentados.

\section{REFERENCIAS BIBLIOGRÁFICAS}

Agamez, E.Y., R.I. Zapata, L.E. Oviedo y J.L. Barrera. 2008. Evaluación de sustratos y procesos de fermentación sólida para la producción de esporas de Trichoderma sp. Rev. Colomb. Biotecnol. 10(2), 23-34.

Agronet. 2015. Zanahoria, resultado de las evaluaciones agropecuarias municipales. En: http://www. agronet.gov.co/Documents/Zanahoria\%20-\%20A\%C3\%B1o\%202015.pdf; consulta: marzo de 2017.

Aranda, E.M., L.E. Georgana, J.A. Ramos y S. Salgado. 2012. Elaboración de un alimento basado en caña de azúcar a partir de la fermentación en estado sólido y con diferentes niveles de zeolitas. Rev. Cubana Cienc. Agríc. 46(2), 159-163.

Biz, A., A.T. Finkler, L. Pitol, B. Medina, N. Krieger y D.A. Mitchell. 2016. Production of pectinases by solid-state fermentation of a mixture of citrus waste and sugarcane bagasse in a pilot-scale packed-bed bioreactor. Biochem. Eng. J. 111(1), 54-62. Doi: 10.1016/j. bej.2016.03.007

Borras-Sandoval, L.M., A.E. Iglesias y G.F. Saavedra-Montañéz. 2015. Evaluación de la dinámica de conservación del producto final de un alimento obtenido por fermentación en estado sólido de la papa (Fes-papa). Cienc. Agric. 12(1), 73-82. Doi: 10.19053/01228420.4125

Borras-Sandoval, L.M., A.E. Iglesias y M.A. Moyano-Bautista. 2014. Efecto de la temperatura y el tiempo sobre los indicadores de la papa (Solanum tuberosum) fermentada en estado sólido. Cienc. Agric. 11(2), 31-38. Doi: 10.19053/01228420.3835

Brea-Maure, O., A. Elías-Iglesias, A. Ortiz-Milán, W. Motta-Ferreira y S. Hechavarría-Riviaux. 2015. Efecto de la urea y del tiempo en la fermentación en estado sólido de la harina de frutos del árbol del pan (Artocarpus altilis). Rev. Cien. Agri. 12(2), 91-101. Doi: 10.19053/01228420.4395

Carrasco, T., A. Ibarra, Y. García, E. Valiño, y T. Pérez. 2003. Efecto negativo de la humedad en la fermentación en estado sólido del bagazo de caña de azúcar. Rev. Cubana Cienc. Avíc. 37(1), 37-41.
Di Marco, O. 2011. Estimación de calidad de los forrajes. Producir XXI 20, 24-30.

Díaz, B., A. Iglesias y E. Valiño. 2014. Impacto de la biotecnología convencional en la seguridad alimentaria a través de producción animal en Ecuador. Estudio de caso: producto BIORÉS. Rev. Int. Cienc. Soc. 1(2), 51-61.

Díaz-Plascencia, D., C. Rodríguez-Muela, P. Mancillas-Flores, C. Angulo, F. Salvador, O. Ruíz, H.O. Rubio, S. Mena y A. Elías. 2010. Desarrollo de un inoculo con diferentes sustratos mediante fermentación sólida sumergida. Rev. Electrón. Vet. 12(1).

Elías, A., O. Lezcano, P. Lezcano, J. Cordero y L. Quintana. 1990. Reseña descriptiva sobre el desarrollo de una tecnología de enriquecimiento proteínico en la caña de azúcar mediante fermentación solida (Saccharina). Rev. Cubana Cienc. Agríc. 24(1), 1-12.

Hernández, S. 2010. Importancia de la fibra en la alimentación de los bovinos. Tesis de pregrado, Facultad de Medicina Veterinaria y Zootecnia, Universidad Michoacana de San Nicolás de Hidalgo, Morelia, México.

Madigan, M.T., J.M. Martinko, J. Parker, T.D. Brock, C. Rodríguez, y M. Sánchez. 2004. Brock biología de los microorganismos. Pearson Educación, México, DF.

Moyano, M.A. 2014. Fermentación en estado sólido (FES) de la papa (Solanum tuberosum), como alternativa tecnológica para la alimentación animal. Tesis de especialista. Escuela de Ciencias Agrícolas, Pecuarias y del Medio Ambiente, Universidad Nacional Abierta y a Distancia, Tunja, Colombia.

Ortega, R. y J.L. Hoyos. 2016. Residuos piscícolas a ensilaje biológico: evaluación fisicoquímica. Publ. Investig. 10(1), 13-20. Doi: 10.22490/25394088.1584

Ramos, J.A., A. Elías y F. Herrera. 2006. Procesos para la producción de un alimento energético-proteico para animales. Efecto de cuatro fuentes energéticas en la fermentación en estado sólido (FES) de la caña de azúcar. Rev. Cubana Cien. Agric. 40(1), 51-58.

Rodríguez, Z., R. Boucourt, A. Elías y M. Madera. 2001. Dinámica de fermentación de mezclas de caña (Saccharum officinarum) y boniato (Ipomea batata). Rev. Cubana Cienc. Agríc. 35(2), 147-151.

Serna, L.F. y S.M. López. 2010. Actualización del manual del laboratorio de análisis de alimentos del programa de tecnología química de la Universidad Tecnológica de Pereira. Tesis en tecnología. Facultad de Tecnología, Universidad Tecnológica de Pereira, Colombia.

Van Soest, P.J., J.B. Robertson y B.A. Lewis. 1991. Methods for dietary fiber, neutral detergent fiber, and nonstarch polysaccharides in relation to animal nutrition. J. Dairy Sci. 74 (1), 3583-3597. Doi: 10.3168/jds. S0022-0302(91)78551-2 